\title{
The Association of Dipyridamole Side Effects with Hemodynamic Parameters, ECG Findings, and Scintigraphy Outcomes
}

\author{
Hamid Javadi ${ }^{1}$, Malihnaz Shariati ${ }^{1}$, Mehdi Mogharrabi ${ }^{1}$, Isa Neshandar Asli ${ }^{2}$, Sara Jallalat ${ }^{1}$, Aref Hooman ${ }^{2}$, \\ Mohammad Seyedabadi ${ }^{3}$, and Majid Assadi ${ }^{3}$ \\ ${ }^{1}$ Department of Nuclear Medicine, 5th Azar Hospital, Golestan University of Medical Science, Gorgan, Iran; ${ }^{2}$ Department of Nuclear \\ Medicine, Taleghani Hospital, Shaheed Beheshti University of Medical Science, Tehran, Iran; and ${ }^{3}$ Bushehr Research Center for \\ Nuclear Medicine, the Persian Gulf Biomedical Sciences Institute, Bushehr University of Medical Sciences, Bushehr, Iran
}

\begin{abstract}
Dipyridamole has extensively been administered as a substitute for physical activity in cardiovascular assessment. The aim of this study was to evaluate the association of dipyridamole side effects with hemodynamic oscillations, scanning results, and electrocardiography (ECG) findings. Methods: Overall, 590 patients referred to the nuclear medicine center for myocardial perfusion imaging were evaluated for adverse dipyridamole effects concurrent with a low level of exercise. Before and during dipyridamole infusion, the patients' vital signs, electrocardiogram, heart rate, systolic blood pressure, and diastolic blood pressure were monitored; all patients underwent stress-rest 99mTc-sestamibi gated SPECT using a 2-d protocol. Results: Eighty-eight patients (14.9\%) experienced at least one side effect during dipyridamole infusion, and abnormal ECG and scan results were observed in $32.4 \%$ and $48.6 \%$ of patients, respectively. We observed a positive correlation between a higher incidence of chest discomfort, headache, and dyspnea and abnormalities on ECG and myocardial perfusion imaging. In addition, these 3 side effects were also associated with a higher postinfusion heart rate, lower preinfusion systolic blood pressure, and lower postinfusion diastolic blood pressure. There were significant differences between pre- and postinfusion heart rate, preinfusion systolic blood pressure, and postinfusion diastolic blood pressure among patients with or without side effects, whereas no significant difference was observed in any of the hemodynamic parameters between patients with normal and abnormal ECG results or myocardial perfusion imaging results. Conclusion: This study demonstrated a correlation between hemodynamic variables and side effects but not with imaging findings or ECG findings.
\end{abstract}

Key Words: dipyridamole; hemodynamic changes; myocardial SPECT; ECG

J Nucl Med Technol 2010; 38:149-152

DOI: 10.2967/jnmt.109.072629

\footnotetext{
Received Nov. 9, 2009; revision accepted Mar. 29, 2010.

For correspondence or reprints contact: Majid Assadi, Bushehr Research Center for Nuclear Medicine, Persian Gulf Biomedical Sciences Institute,

Boostan 19 Alley, Imam Khomeini St., Bushehr 75147, Iran.

E-mail: assadipoya@yahoo.com, asadi@bpums.ac.ir

COPYRIGHT @ 2010 by the Society of Nuclear Medicine, Inc.
}

$\mathbf{T}$ he administration of dipyridamole during perfusion scintigraphy has extensively been applied to simulate the effects of physical activity in patients who are incapable of performing a conventional exercise test (1-3). The addition of a symptom-limited exercise test is also considered safe and efficient. Compared with dipyridamole infusion alone, exercise added to dipyridamole infusion has been shown to result in fewer noncardiac side effects, the same prevalence of angina pectoris, and a higher incidence of ST segment changes (4).

Dipyridamole, by itself, can cause hypotension, dyspnea, dizziness, and electrocardiography (ECG) abnormalities; however, simultaneous dipyridamole administration and scanning should be treated as a distinct situation and demands its own risk assessment. Several studies have evaluated the safety and diagnostic precision of dipyridamole-thallium imaging in subjects with suspected coronary artery disease $(5,6)$.

The purpose of this study was to assess the incidence of dipyridamole side effects and their association with hemodynamic changes, scanning results, and ECG findings.

\section{MATERIALS AND METHODS}

\section{Participants and Study Design}

Overall, 590 patients (mean age $\pm \mathrm{SD}, 57 \pm 11$ y) who underwent myocardial perfusion scanning with intravenous dipyridamole in the stress phase for detection of possible coronary artery disease were assessed in a prospective study. The patients fasted overnight, and all cardiovascular medications were discontinued at least $2 \mathrm{~d}$ before the study. The ECG, blood pressure, heart rate, systolic blood pressure, and diastolic blood pressure of all patients were determined both before and after dipyridamole administration. Infusion of dipyridamole $(0.568 \mathrm{mg} / \mathrm{kg})$ was followed by a low level of exercise $(60 \mathrm{rpm} / 30 \mathrm{~W})$ for $3 \mathrm{~min}$ to attain maximal coronary blood flow and to reduce radionuclide uptake in the gastrointestinal organs (4). Four minutes later, 
99m Tc-sestamibi (740 MBq) was administered parenterally and imaging was performed 90 min after the initial infusion of dipyridamole; the rest-phase examination was performed on the following day. An abnormal ECG was designated as one showing a $0.1-\mathrm{mV}$ horizontal or downsloping ST segment depression of $80 \mathrm{~ms}$ after the $\mathrm{J}$ point.

\section{Acquisition Protocol}

All patients underwent stress-rest ${ }^{99 \mathrm{~m}} \mathrm{Tc}$-sestamibi gated SPECT using a 2-d protocol. Rest gated SPECT was performed $90 \mathrm{~min}$ after intravenous injection of $740 \mathrm{MBq}$ of 99mTc-sestamibi. SPECT was performed with the patient supine. We used a dual-head $\gamma$-camera in the $90^{\circ}$ setting (DLXi; GE Healthcare), equipped with high-resolution, low-energy collimators. Sixty-four views over $180^{\circ}$ were obtained from $45^{\circ}$ right anterior oblique to $45^{\circ}$ left posterior oblique. Images were acquired for $25 \mathrm{~s}$ per view with a zoom factor of 1.45 , were gated at 8 frames per cardiac cycle using an R-wave trigger, and were stored in a $64 \times$ 64 matrix. The gated short-axis images were then processed for automatic left ventricular ejection fraction quantification using MultiDim quantitative gated SPECT software (Stanford University).

The study was approved by the institutional ethics committee of Golestan University of Medical Science and complied with the tenets of the Declaration of Helsinki; all patients gave written informed consent.

\section{Statistical Analysis}

The data are represented as mean $\pm \mathrm{SD}$; to compare quantitative variables between groups, we used either the $t$ test or the paired $t$ test; the $\chi^{2}$ test was also applied to analyze nonparametric data; a $P$ value of less than 0.05 was considered to be statistically significant.

\section{RESULTS}

Overall, 88 patients (14.9\%) experienced at least one undesirable side effect during dipyridamole infusion; among which chest discomfort (5.2\%) stands as the most frequent complaint (Table 1); an abnormal ECG and irregular scan findings were observed in 191 (32.4\%) and 287 (48.6\%) patients, respectively. Patients with abnormal ECG findings had a remarkably higher incidence of side effects than did patients with normal ECG findings $(32.9 \%$ vs. $6.2 \%$; $P<$ 0.05). The same findings were also observed when we compared the data of patients with abnormal scans to those with normal scans $(22.6 \%$ vs. $7.5 \% ; P<0.05)$. The ejection fraction was $57 \% \pm 12 \%$ in the stress phase and $59 \% \pm 11 \%$ on rest-phase gated images. A higher incidence of chest discomfort, headache, and dyspnea correlated positively with abnormal findings on the ECG and myocardial perfusion images. In addition, these 3 side effects were associated with a higher postinfusion heart rate, lower preinfusion systolic blood pressure, and lower postinfusion diastolic blood pressure.

Dipyridamole administration resulted in a rise in heart rate $(82 \pm 12$ vs. $89 \pm 13$ beats/min) and systolic blood
TABLE 1

Frequency of Various Side Effects in Patients

\begin{tabular}{lc}
\hline \multicolumn{1}{c}{ Side Effect } & $n$ \\
\hline Chest discomfort & $31(5.2 \%)$ \\
Dizziness & $5(0.8 \%)$ \\
Dyspnea & $18(3 \%)$ \\
Flushing & $6(1 \%)$ \\
Headache & $15(2.5 \%)$ \\
Nausea & $3(0.5 \%)$ \\
Palpitation & $3(0.5 \%)$ \\
Sweating & $4(0.6 \%)$ \\
Ventricular tachycardia & $1(0.1 \%)$ \\
Vomiting & $2(0.3 \%)$ \\
Total & $88(14.9 \%)$ \\
\hline
\end{tabular}

pressure $(138 \pm 20$ vs. $132 \pm 21 \mathrm{~mm} \mathrm{Hg})$ and a fall in diastolic blood pressure $(79 \pm 8$ vs. $75 \pm 9 \mathrm{~mm} \mathrm{Hg})(P<$ 0.001). Comparing the data of patients who experienced at least one side effect with those who had no side effects, we found a significantly higher postinfusion heart rate (82 \pm 13 vs. $92 \pm 10$ beats/min; $P=0.016)$, a lower preinfusion systolic blood pressure $(139 \pm 20$ vs. $133 \pm$ $23 \mathrm{~mm} \mathrm{Hg} ; P=0.024)$, and a lower postinfusion diastolic blood pressure $(76 \pm 9$ vs. $72 \pm 10 \mathrm{~mm} \mathrm{Hg} ; P=0.003)$ in patients who had side effects. However, there were no noticeable alterations in resting heart rate, preinfusion diastolic blood pressure, and postinfusion systolic blood pressure $(P>0.05)$ (Table 2$)$.

No significant difference was observed in any of the hemodynamic parameters when patients with an abnormal ECG were compared with those with normal ECG findings; the same results were also obtained between normal and abnormal myocardial perfusion imaging groups $(P>0.05)$ (Table 2).

\section{DISCUSSION}

Intravenous dipyridamole administration during perfusion scintigraphy is applied as an alternative to exercise for coronary artery disease detection and risk assessment in clinical practice. Dipyridamole enhances left ventricular ejection fraction and heart rate and slightly diminishes diastolic blood pressure in individuals with a lower possibility of having coronary artery disease (7); on the other hand, poststress left ventricular ejection fraction decreases significantly in patients with ischemia $(3,8)$ because of end-systolic ventricular dilation $(9,10)$ and inadequate ventricular contraction $(11,12)$. We detected a decrease in systolic blood pressure, diastolic blood pressure, and left ventricular ejection fraction after dipyridamole administration unrelated to the existence of side effects or abnormalities in ECG and scintigraphy scans.

We observed an increment in heart rate and a decrement in systolic blood pressure after dipyridamole infusion. Homma et al. (5) and Ignaszewski et al. (13), in 2 separate studies, have also reported similar results with both nonexercise and symptom-limited exercise scintigraphy with dipyridamole. 
TABLE 2

Hemodynamic Parameters Before and After Dipyridamole with Respect to Side Effects, ECG Findings, and Scan Findings

\begin{tabular}{|c|c|c|c|c|c|c|c|c|c|}
\hline \multirow[b]{2}{*}{ Parameter } & \multirow[b]{2}{*}{ Category } & \multicolumn{2}{|c|}{ Heart rate } & \multicolumn{2}{|c|}{ Systolic blood pressure } & \multicolumn{2}{|c|}{$\begin{array}{l}\text { Diastolic blood } \\
\text { pressure }\end{array}$} & \multicolumn{2}{|c|}{ Ejection fraction } \\
\hline & & Before & After & Before & After & Before & After & Before & After \\
\hline \multirow[t]{2}{*}{ Side effects } & No & $82 \pm 12$ & $82 \pm 13$ & $139 \pm 20$ & $132 \pm 20$ & $79 \pm 8$ & $76 \pm 9$ & $60 \pm 11$ & $58 \pm 12$ \\
\hline & Yes & $82 \pm 10$ & $92 \pm 10^{*}$ & $133 \pm 23^{*}$ & $131 \pm 23$ & $79 \pm 10$ & $72 \pm 10^{*}$ & $53 \pm 14$ & $50 \pm 14$ \\
\hline \multirow[t]{2}{*}{ ECG findings } & Normal & $82 \pm 12$ & $88 \pm 13$ & $139 \pm 21$ & $132 \pm 20$ & $79 \pm 8$ & $75 \pm 9$ & $64 \pm 8$ & $63 \pm 8$ \\
\hline & Abnormal & $82 \pm 11$ & $90 \pm 12$ & $137 \pm 20$ & $131 \pm 20$ & $79 \pm 9$ & $75 \pm 10$ & $47 \pm 13$ & $44 \pm 13$ \\
\hline \multirow[t]{2}{*}{ Scan findings } & Normal & $77 \pm 8$ & $82 \pm 8$ & $138 \pm 22$ & $131 \pm 21$ & $79 \pm 8$ & $75 \pm 9$ & $66 \pm 7$ & $65 \pm 7$ \\
\hline & Abnormal & $88 \pm 13$ & $96 \pm 13$ & $138 \pm 20$ & $133 \pm 19$ & $78 \pm 9$ & $75 \pm 9$ & $50 \pm 13$ & $49 \pm 14$ \\
\hline
\end{tabular}

${ }^{\star} P<0.05$.

Data are mean $\pm S D$.

A retrospective investigation on 933 subjects has revealed that patients who exhibited some form of untoward reaction to dipyridamole were $10 \%$ more liable to show an abnormal perfusion examination outcome (14). Meyers et al. have also described headache as being the most prevalent adverse effect of dipyridamole in patients undergoing dual-isotope ${ }^{201} \mathrm{Tl}$ rest/ ${ }^{99 \mathrm{~m}} \mathrm{Tc}$-sestamibi stress imaging (15). Laarman et al. have shown that low-level exercise during dipyridamole infusion causes fewer noncardiac side effects, a similar incidence of angina, and a superior frequency of ST-segment changes, compared with dipyridamole alone (4). Our study did not contain a group without exercise; however, noncardiac side effects $(13 \%)$ were less frequent and ECG abnormalities $(32.3 \%)$ were more common than in similar studies without exercise.

In a study on 3,911 patients to evaluate the mortality and morbidity of dipyridamole-thallium imaging, Ranhosky et al. reported 2 deaths due to myocardial infarction and 2 additional nonfatal myocardial infarctions, 6 subjects with acute bronchospasm, and the following percentages of patients with additional side effects: chest pain (19.7\%), headache $(12.2 \%)$, dizziness $(11.8 \%)$, and ST-T changes (7.5\%) (16).

We also found a positive correlation between having abnormal ECG or scintigraphy findings and the likelihood of exhibiting dipyridamole side effects, especially chest discomfort, headache, and dyspnea. A similar correlation has also been stated by Villanueva et al. between ST-segment depression and chest pain or ECG results and redistribution defects (17).

Furthermore, our study revealed no significant difference between peripheral hemodynamic response and central coronary defects, confirming the previous finding of Zhu et al (18). This finding may indicate that defects revealed by dipyridamole are important even when there is no distortion of peripheral hemodynamic response. We have categorized patients only as having normal or abnormal ECG and scintigraphy outcomes, and we did not additionally study the correlation of side effects and hemodynamic parameters with different types of ECG or imaging abnormalities - a topic that demands further investigation.

\section{CONCLUSION}

This study demonstrated a correlation between hemodynamic variables and side effects but not with imaging findings or ECG findings.

\section{ACKNOWLEDGMENTS}

This study was performed under the sponsorship of Golestan University of Medical Sciences (grant 393). Thanks are extended to the colleagues at our institutes for technical help and data acquisition.

\section{REFERENCES}

1. Leppo JA. Dipyridamole myocardial perfusion imaging. J Nucl Med. 1994;35:730-733.

2. Candell-Riera J, Santana-Boado C, Castell-Conesa J, et al. Simultaneous dipyridamole/maximal subjective exercise with ${ }^{99 \mathrm{~m}} \mathrm{Tc}-\mathrm{MIBI}$ SPECT: improved diagnostic yield in coronary artery disease. J Am Coll Cardiol. 1997;29:531-536

3. Zafrir N, Bassevitch R, Shimoni A, Teplitsky I, Lubin E. Effect of dipyridamole on myocardial perfusion and function using technetium-99m MIBI. Int $J$ Cardiol. 1995;49:25-31.

4. Laarman GJ, Niemeyer MG, van der Wall EE, et al. Dipyridamole thallium testing: noncardiac side effects, cardiac effects, electrocardiographic changes and hemodynamic changes after dipyridamole infusion with and without exercise. Int J Cardiol. 1988;20:231-238.

5. Homma S, Gilliland Y, Guiney TE, Strauss HW, Boucher CA. Safety of intravenous dipyridamole for stress testing with thallium imaging. Am J Cardiol. 1987;59:152-154.

6. Perper EJ, Segall GM. Safety of dipyridamole-thallium imaging in high risk patients with known or suspected coronary artery disease. J Nucl Med. 1991;32:2107-2114.

7. Weinmann P, Moretti JL. Effects of dipyridamole on left ventricular function. J Nucl Cardiol. 2000;7:103-106.

8. Ben-Haim S, Gips S, Merdler A, Front A, Tamir A. Myocardial stunning demonstrated with rest and post-stress measurements of left ventricular function using dual-isotope gated myocardial perfusion SPECT. Nucl Med Commun. 2004;25:657-663.

9. Bestetti A, Di Leo C, Alessi A, Triulzi A, Tagliabue L, Tarolo GL. Post-stress end-systolic left ventricular dilation: a marker of endocardial post-ischemic stunning. Nucl Med Commun. 2001;22:685-693.

10. Bestetti A, Scalzi PB, Alessi A, et al. Detection and assessment of myocardial stunning with perfusion tomographic scintigraphy using gated SPECT [in Italian]. Ital Heart J Suppl. 2000;1:790-796. 
11. Mut F, Beretta M, Vidal I. Identification of myocardial stunning by means of gated perfusion SPECT in patients undergoing ischaemic stress myocardial tests. World J Nucl Med. 2003;2:122-125.

12. Dabbagh Kakhki VR, Jabari H. Dipyridamole stress and rest gated ${ }^{99 \mathrm{~m}} \mathrm{Tc}-$ sestamibi myocardial perfusion SPECT: left ventricular function indices and myocardial perfusion findings. Iran J Nucl Med. 2007;15:1-7.

13. Ignaszewski AP, McCormick LX, Heslip PG, McEwan AJ, Humen DP. Safety and clinical utility of combined intravenous dipyridamole/ symptom-limited exercise stress test with thallium-201 imaging in patients with known or suspected coronary artery disease. J Nucl Med. 1993;34: 2053-2061.

14. Meyers A, Topham L, Ballow J, Totah D, Wilke R. Age, perfusion test results and dipyridamole reaction. Radiol Technol. 2002;73:409-414.
15. Meyers AM, Topham L, Ballow J, Totah D, Wilke R. Adverse reactions to dipyridamole in patients undergoing stress/rest cardiac perfusion testing. $J$ Nucl Med Technol. 2002;30:21-24.

16. Ranhosky A, Kempthorne-Rawson J. The safety of intravenous dipyridamole thallium myocardial perfusion imaging. Intravenous Dipyridamole Thallium Imaging Study Group. Circulation. 1990;81:12051209.

17. Villanueva FS, Smith WH, Watson DD, Beller GA. ST-segment depression during dipyridamole infusion, and its clinical, scintigraphic and hemodynamic correlates. Am J Cardiol. 1992;69:445-448.

18. Zhu YY, Lee W, Botvinick E, et al. The clinical and pathophysiologic implications of pain, ST abnormalities, and scintigraphic changes induced during dipyridamole infusion: their relationships to the peripheral hemodynamic response. Am Heart J. 1988;116:1071-1080. 